\title{
(c) creative
}

ISSN 1855-3966 (printed edn.), ISSN 1855-3974 (electronic edn.)

\author{
ARS MATHEMATICA CONTEMPORANEA 21 (2021) \#P1.08 \\ https://doi.org/10.26493/1855-3974.2450.1dc \\ (Also available at http://amc-journal.eu)
}

\section{On 2-closures of rank 3 groups}

\author{
Saveliy V. Skresanov * \\ Sobolev Institute of Mathematics, 4 Acad. Koptyug avenue, 630090, Novosibirsk, Russia \\ Novosibirsk State University, 1 Pirogova street, 630090 Novosibirsk, Russia
}

Received 29 September 2020, accepted 2 April 2021, published online 23 August 2021

\begin{abstract}
A permutation group $G$ on $\Omega$ is called a rank 3 group if it has precisely three orbits in its induced action on $\Omega \times \Omega$. The largest permutation group on $\Omega$ having the same orbits as $G$ on $\Omega \times \Omega$ is called the 2-closure of $G$. A description of 2-closures of rank 3 groups is given. As a special case, it is proved that the 2-closure of a primitive one-dimensional affine rank 3 group of sufficiently large degree is also affine and one-dimensional.
\end{abstract}

Keywords: 2-closure, rank 3 group, rank 3 graph, permutation group.

Math. Subj. Class. (2020): 20B25, 20B15, 05E18

\section{Introduction}

Let $G$ be a permutation group on a finite set $\Omega$. Recall that the rank of $G$ is the number of orbits in the induced action of $G$ on $\Omega \times \Omega$; these orbits are called 2-orbits. If a rank 3 group has even order, then its non-diagonal 2-orbit induces a strongly regular graph on $\Omega$, which is called a rank 3 graph. It is readily seen that a rank 3 group acts on the corresponding rank 3 graph as an automorphism group. Notice that an arc-transitive strongly regular graph need not be a rank 3 graph, since its automorphism group might be intransitive on non-arcs.

Related to this is the notion of a 2-closure of a permutation group [31]. The 2-closure $G^{(2)}$ of a permutation group $G$ is the largest permutation group having the same 2-orbits as $G$. Clearly $G \leq G^{(2)}$, the 2-closure of $G^{(2)}$ is equal to $G^{(2)}$, and $G^{(2)}$ has the same rank as $G$. Note also that given a rank 3 graph $\Gamma$ corresponding to the rank 3 group $G$, we have $\operatorname{Aut}(\Gamma)=G^{(2)}$.

The rank 3 groups are completely classified. A primitive rank 3 group either stabilizes a nontrivial product decomposition, or is almost simple or is an affine group. The rank 3 groups stabilizing a nontrivial product decomposition are given by the classification of the

${ }^{*}$ The work is supported by Mathematical Center in Akademgorodok under agreement No. 075-15-2019-1613 with the Ministry of Science and Higher Education of the Russian Federation.

E-mail address: skresan@math.nsc.ru (Saveliy V. Skresanov) 
2-transitive almost simple groups, see Theorem 4.1 (ii)(a) and Section 5 in [6]. Almost simple rank 3 groups were determined in [1] when the socle is an alternating group, in [16] when the socle is a classical group and in [21] when the socle is an exceptional or sporadic group. The classification of affine rank 3 groups was completed in [19].

In order to describe the 2-closures of rank 3 groups (or, equivalently, the automorphism groups of rank 3 graphs), it is essential to know which rank 3 groups give rise to isomorphic graphs. Despite all the rank 3 groups being known, it is not a trivial task (considerable progress in describing rank 3 graphs was made in [4]). In the present paper we give a detailed description of the 2-closures of rank 3 groups.

Theorem 1.1. Let $G$ be a rank 3 permutation group on a set $\Omega$. Then either $G$ is one of the groups from Table 8, or exactly one of the following is true.

(i) $G$ is imprimitive, i.e. it preserves a nontrivial decomposition $\Omega=\Delta \times X$. Then $G^{(2)}=\operatorname{Sym}(\Delta) \imath \operatorname{Sym}(X)$.

(ii) $G$ is primitive and preserves a product decomposition $\Omega=\Delta^{2}$. Then $G^{(2)}=$ $\operatorname{Sym}(\Delta) \uparrow \operatorname{Sym}(2)$.

(iii) $G$ is primitive almost simple with socle $L$, i.e. $L \unlhd G \leq \operatorname{Aut}(L)$. Then $G^{(2)}=$ $N_{\operatorname{Sym}(\Omega)}(L)$, and $G^{(2)}$ is almost simple with socle $L$.

(iv) $G$ is a primitive affine group which does not stabilize a product decomposition. Then $G^{(2)}$ is also an affine group. More precisely, there exist an integer $a \geq 1$ and a prime power $q$ such that $G \leq \mathrm{A \Gamma L}_{a}(q)$, and exactly one of the following holds (setting $F=\mathrm{GF}(q))$.

(a) $G \leq \operatorname{A\Gamma L}_{1}(q)$. Then $G^{(2)} \leq \operatorname{A\Gamma L}_{1}(q)$.

(b) $G \leq \mathrm{A \Gamma L}_{2 m}(q)$ preserves the bilinear forms graph $H_{q}(2, m), m \geq 3$. Then

$$
G^{(2)}=F^{2 m} \rtimes\left(\left(\mathrm{GL}_{2}(q) \circ \mathrm{GL}_{m}(q)\right) \rtimes \operatorname{Aut}(F)\right) .
$$

(c) $G \leq \mathrm{A}_{2 m}(q)$ preserves the affine polar graph $\mathrm{VO}_{2 m}^{\epsilon}(q), m \geq 2, \epsilon= \pm$. Then

$$
G^{(2)}=F^{2 m} \rtimes \Gamma \mathrm{O}_{2 m}^{\epsilon}(q) .
$$

(d) $G \leq \mathrm{A \Gamma L}_{10}(q)$ preserves the alternating forms graph $A(5, q)$. Then

$$
G^{(2)}=F^{10} \rtimes\left(\left(\Gamma L_{5}(q) /\{ \pm 1\}\right) \times\left(F^{\times} /\left(F^{\times}\right)^{2}\right)\right) .
$$

(e) $G \leq \mathrm{A \Gamma L}_{16}(q)$ preserves the affine half spin graph $\mathrm{VD}_{5,5}(q)$. Then $G^{(2)} \leq$ $\mathrm{A \Gamma L}_{16}(q)$ and

$$
G^{(2)}=F^{16} \rtimes\left(\left(F^{\times} \circ \operatorname{Inndiag}\left(D_{5}(q)\right)\right) \rtimes \operatorname{Aut}(F)\right) .
$$

(f) $G \leq \mathrm{A \Gamma L}_{4}(q)$ preserves the Suzuki-Tits ovoid graph $\operatorname{VSz}(q), q=2^{2 e+1}$, $e \geq 1$. Then

$$
G^{(2)}=F^{4} \rtimes\left(\left(F^{\times} \times \operatorname{Sz}(q)\right) \rtimes \operatorname{Aut}(F)\right) .
$$


Up arrow symbol in (ii) denotes the primitive wreath product (see Section 2), notation for graphs in the affine case is explained in Section 3. Table 8 contains finitely many permutation groups and can be found in Appendix. We note that the largest degree of a permutation group from Table 8 is $3^{12}$.

We also remark that the value of $a$ in (iv) of Theorem 1.1 is not necessarily minimal subject to $G \leq \mathrm{A \Gamma L}_{a}(q)$, since it is not completely defined by the corresponding rank 3 graph and may depend on the group-theoretical structure of $G$. Minimal values of $a$ can be found in Table 1.

The proof of Theorem 1.1 can be divided into three parts. First we reduce the study to the case when $G^{(2)}$ has the same socle as $G$, and deal with cases (i)-(iii) (Proposition 2.8). In the affine case (iv) we apply the classification of affine rank 3 groups [19], and compare subdegrees of groups from various classes (Lemma 3.5 and Proposition 3.6); that allows us to deal with case (a). Finally, we invoke known results on automorphisms of some families of strongly regular graphs to cover cases (b)-(d), while cases (e) and (f) are treated separately.

The case (iv), (a) of Theorem 1.1 can be formulated as a standalone result that may be of the independent interest.

Theorem 1.2. Let $G$ be a primitive affine permutation group of rank 3 and suppose that $G \leq \mathrm{A \Gamma L}_{1}(q)$ for some prime power $q$. Then $G^{(2)} \leq \mathrm{A \Gamma L}_{1}(q)$, unless the degree and the smallest subdegree of $G$ are as in Table 7.

It is important to stress that the group $G$ in Theorem 1.2 can have a nonsolvable 2closure; such an example of degree $2^{6}$ has been found in [28].

The main motivation for the present study is the application of Theorem 1.1 to the computational 2-closure problem. Namely, the problem asks if given generators of a rank 3 group one can find generators of its 2-closure in polynomial time. This task influenced the scope of the present paper considerably, for instance, while one can determine the structure of the normalizer in Theorem 1.1 (iii) explicitly depending on the type of the corresponding rank 3 graph, this is not required for the computational problem as this normalizer can be computed in polynomial time [22]. In other cases it is possible to compute automorphism groups of associated rank 3 graphs directly (for example, of Hamming graphs), but in many situations a more detailed study of relevant groups is required. The author plans to turn to the computational problem in his future work.

Finally, the author would like to express his gratitude to professors M. Grechkoseeva, I. Ponomarenko, A. Vasil'ev and to the anonymous referees for numerous helpful comments and suggestions.

\section{Reduction to affine case}

We will prove Theorem 1.1 by dealing with rank 3 groups on a case by case basis. Recall the following well-known general classification of rank 3 groups.

Proposition 2.1. Let $G$ be a rank 3 group with socle $L$. Then $G$ is transitive and one of the following holds:

(i) $G$ is imprimitive,

(ii) $L$ is a direct product of two isomorphic simple groups, and $G$ preserves a nontrivial product decomposition, 
(iii) L is nonabelian simple,

(iv) L is elementary abelian.

Proof. Transitivity part is clear. If $G$ is primitive, Theorem 4.1 and Proposition 5.1 from [6] imply that $G$ belongs to one of the last three cases from the statement.

Suppose that $G \leq \operatorname{Sym}(\Omega)$. Observe that $G$ acts imprimitively on $\Omega$ if and only if it preserves a nontrivial decomposition $\Omega=\Delta \times X$, i.e. the action domain $\Omega$ can be identified with a nontrivial Cartesian product $\Delta \times X,|\Delta|>1,|X|>1$, where $G$ permutes blocks of the form $\Delta \times\{x\}, x \in X$. Denote by $\operatorname{Sym}(\Delta)$ i $\operatorname{Sym}(X) \leq \operatorname{Sym}(\Omega)$ the wreath product of $\operatorname{Sym}(\Delta)$ and $\operatorname{Sym}(X)$ in the imprimitive action, so $G \leq \operatorname{Sym}(\Delta)$ i $\operatorname{Sym}(X)$.

Proposition 2.2. Let $G$ be an imprimitive rank 3 permutation group on $\Omega$. Let $\Delta$ be $a$ nontrivial block of imprimitivity of $G$, so $\Omega$ can be identified with $\Delta \times X$ for some set $X$. Then $G^{(2)}=\operatorname{Sym}(\Delta)$ ¿ $\operatorname{Sym}(X)$.

Proof. Set $H=\operatorname{Sym}(\Delta) \imath \operatorname{Sym}(X)$. Then $G \leq H$ and since $G$ and $H$ are both groups of rank 3, we have $G^{(2)}=H^{(2)}$. By [15, Lemma 2.5] (see also [8, Proposition 3.1]), we have

$$
(\operatorname{Sym}(\Delta) \imath \operatorname{Sym}(X))^{(2)}=\operatorname{Sym}(\Delta)^{(2)} \prec \operatorname{Sym}(X)^{(2)}=\operatorname{Sym}(\Delta) \prec \operatorname{Sym}(X),
$$

so $H$ is 2-closed. Hence $G^{(2)}=H^{(2)}=H$, as claimed.

Suppose that the action domain is a Cartesian power of some set: $\Omega=\Delta^{m}, m \geq 2$ and $|\Delta|>1$. Denote by $\operatorname{Sym}(\Delta) \uparrow \operatorname{Sym}(m)$ the wreath product of $\operatorname{Sym}(\Delta)$ and $\operatorname{Sym}(m)$ in the product action, i.e. the base group acts on $\Delta^{m}$ coordinatewise, while $\operatorname{Sym}(m)$ permutes the coordinates. We say that $G \leq \operatorname{Sym}(\Omega)$ preserves a nontrivial product decomposition $\Omega=\Delta^{m}$ if $G \leq \operatorname{Sym}(\Delta) \uparrow \operatorname{Sym}(m)$.

If $G$ preserves a nontrivial product decomposition $\Omega=\Delta^{m}$, then $G$ induces a permutation group $G_{0} \leq \operatorname{Sym}(\Delta)$. Recall that we can identify $G$ with a subgroup of $G_{0} \uparrow$ $\operatorname{Sym}(m)$. We need the following well-known formula for the rank of a primitive wreath product; the proof is provided for completeness (see also [18]).

Lemma 2.3. Let $G$ be a transitive group of rank $r$. Then $G \uparrow \operatorname{Sym}(m)$ has rank $\left(\begin{array}{c}r+m-1 \\ m\end{array}\right)$.

Proof. Let $G \leq \operatorname{Sym}(\Delta)$, and recall that $\Gamma=G \uparrow \operatorname{Sym}(m)$ acts on $\Delta^{m}$. Choose $\alpha_{1} \in \Delta$ and set $\overline{\alpha_{1}}=\left(\alpha_{1}, \ldots, \alpha_{1}\right) \in \Delta^{m}$. Let $\alpha_{1}, \ldots, \alpha_{r}$ be representatives of orbits of $G_{\alpha_{1}}$ on $\Delta$. Since the point stabilizer $\Gamma_{\overline{\alpha_{1}}}$ is equal to $G_{\alpha_{1}} \uparrow \operatorname{Sym}(m)$, the points $\left(\alpha_{i_{1}}, \ldots, \alpha_{i_{m}}\right)$, $1 \leq i_{1} \leq \cdots \leq i_{m} \leq r$, form a set of representatives of orbits of $\Gamma_{\overline{\alpha_{1}}}$ on $\Delta^{m}$. The number of indices $i_{1}, \ldots, i_{m}$ satisfying $1 \leq i_{1} \leq \cdots \leq i_{m} \leq r$ is equal to the number of weak compositions of $m$ into $r$ parts, hence the claim is proved.

Observe that in the particular case when $\Omega=\Delta^{2}$, the wreath product $\operatorname{Sym}(\Delta) \uparrow$ $\operatorname{Sym}(2)$ has rank 3 and its 2-orbit of size $|\Delta|^{2}(|\Delta|-1)$ is the edge set of the Hamming graph $H(2,|\Delta|)$.

Proposition 2.4. Let $G$ be a primitive rank 3 permutation group on $\Omega$, preserving a nontrivial product decomposition $\Omega=\Delta^{m}, m \geq 2$. Then $m=2$, a 2-orbit of $G$ induces $a$ Hamming graph and $G^{(2)}=\operatorname{Sym}(\Delta) \uparrow \operatorname{Sym}(2)$. 
Proof. Set $H=\operatorname{Sym}(\Delta) \uparrow \operatorname{Sym}(m)$, and recall that by Lemma 2.3, $H$ has $\operatorname{rank}\left(\begin{array}{c}2+m-1 \\ m\end{array}\right)=$ $m+1$ as a permutation group. Since $G \leq H$, we have $m+1 \leq 3$. Therefore $m=2$ and $H$ is a rank 3 group. Then $G^{(2)}=H^{(2)}$ and it suffices to show that $H$ is 2-closed.

A 2-orbit of $H$ induces the Hamming graph $H(2, q)$ on $\Omega$, where $q=|\Delta|$. By [3, Theorem 9.2.1], $\operatorname{Aut}(H(2, q))=\operatorname{Sym}(q) \uparrow \operatorname{Sym}(2)$. It readily follows that $H^{(2)}=$ $\operatorname{Aut}(H(2, q))=H$, completing the proof.

In order to find 2-closures in the last two cases of Proposition 2.1, we need to show that 2-closure almost always preserves the socle of a rank 3 group.

Lemma 2.5. Let $G$ be a primitive rank 3 group and suppose that $G$ and $G^{(2)}$ have different socles. Then either $G$ preserves a nontrivial product decomposition, or $G$ is an almost simple group with socle and degree as in Table 8.

Proof. From [25, Theorem 2] it follows that either $G$ preserves a nontrivial product decomposition, or $G$ and $G^{(2)}$ are almost simple groups. By [20, Theorem 1], the latter situation applies only to a finite number of rank 3 groups, namely, either $G$ is one of exceptional examples from [20, Table 1], or the socle of $G$ is $G_{2}(q), q \geq 3$, or the socle is $\Omega_{7}(q)$. Since rank 3 graphs are distance-transitive, [20, Proposition 1] implies $q \in\{3,4,8\}$ in the case of $G_{2}(q)$, while [20, Proposition 2] yields $q \in\{2,3\}$ in the case of $\Omega_{7}(q)$.

Lemma 2.6. Let $G$ be a primitive rank 3 group with nonabelian simple socle. Then $G$ does not preserve a nontrivial product decomposition.

Proof. Let $G \leq \operatorname{Sym}(\Omega)$ and $L$ be the socle of $G$. Suppose on the contrary that $G$ preserves a nontrivial product decomposition. Since $G$ is primitive, and $L$ is a nonabelian simple minimal normal subgroup of $G$, [26, Theorem 8.21] implies that either $L$ is $A_{6}$ and $|\Omega|=$ 36 , or $L=M_{12}$ and $|\Omega|=144$, or $L=\operatorname{Sp}_{4}(q), q \geq 4, q$ even and $|\Omega|=q^{4}\left(q^{2}-1\right)^{2}$. One can easily check that neither of these situations occurs in rank 3 by inspecting the classification of almost simple rank 3 groups. The reader is referred to [5, Table 5] for alternating socles, [5, Table 9] for sporadic socles and [5, Tables 6 and 7] for classical socles.

It should be noted that an almost simple group with rank larger than 3 might preserve a nontrivial product decomposition, see [26, Section 1.3].

Proposition 2.7. Let $G$ be a primitive rank 3 permutation group on $\Omega$ with nonabelian simple socle $L$. Then either $G$ appears in Table 8 , or $G^{(2)}$ has socle $L$ and $G^{(2)}=$ $N_{\mathrm{Sym}(\Omega)}(L)$.

Proof. By Lemma 2.6, $G$ does not preserve a nontrivial product decomposition, hence by Lemma 2.5, either $G$ belongs to Table 8, or 2-closure $G^{(2)}$ has the same socle as $G$. Set $N=N_{\text {Sym }(\Omega)}(L)$. Clearly $G^{(2)} \leq N$, and to establish equality it suffices to show that $N$ is a rank 3 group.

Suppose that this not the case and $N$ is 2-transitive. By [6, Proposition 5.2], $N$ has a unique minimal normal subgroup, and since $L$ is a minimal normal subgroup of $N$, the socle of $N$ must be equal to $L$. Hence $N$ is an almost simple 2-transitive group with socle $L$.

The possibilities for a socle of a 2-transitive almost simple group are all known and moreover, such a socle is a 2-transitive group itself, unless $G$ acts on 28 points and $L=$ 
$\mathrm{PSL}_{2}(8)$ (see Theorem 5.3 (S) and the following notes in [6]). By [16, Theorem 1.2], there is no rank 3 group of degree 28 with socle $\mathrm{PSL}_{2}(8)$, hence $L$ and thus $G$ are 2-transitive, which is a contradiction. Therefore $N$ is a rank 3 group and $G^{(2)}=N$.

We summarize the results of this section in the following.

Proposition 2.8. Let $G$ be a rank 3 permutation group on $\Omega$. Then either $G$ appears in Table 8, or exactly one of the following holds.

(i) $G$ is imprimitive, i.e. it preserves a nontrivial decomposition $\Omega=\Delta \times X$. Then $G^{(2)}=\operatorname{Sym}(\Delta)$ i $\operatorname{Sym}(X)$.

(ii) $G$ is primitive and preserves a product decomposition $\Omega=\Delta^{2}$. Then $G^{(2)}=$ $\operatorname{Sym}(\Delta) \uparrow \operatorname{Sym}(2)$.

(iii) $G$ is a primitive almost simple group with socle $L$, i.e. $L \unlhd G \leq \operatorname{Aut}(L)$. Then $G^{(2)}=N_{\operatorname{Sym}(\Omega)}(L)$, and $G^{(2)}$ is almost simple with socle $L$.

(iv) $G$ is a primitive affine group which does not stabilize a product decomposition. Then $G^{(2)}$ is also an affine group.

\section{Affine case}

In the previous section we reduced the task of describing the 2-closures of rank 3 groups to the case when the group in question is affine. Recall that a primitive permutation group $G \leq \operatorname{Sym}(\Omega)$ is called affine, if it has a unique minimal normal subgroup $V$ equal to its socle, such that $V$ is an elementary abelian $p$-group for some prime $p$ and $G=V \rtimes G_{0}$ for some $G_{0}<G$. The permutation domain $\Omega$ can be identified with $V$ in such a way that $V$ acts on it by translations, and $G_{0}$ acts on it as a subgroup of $\operatorname{GL}(V)$. Clearly $G_{0}$ is the stabilizer of the zero vector in $V$ under such identification.

If $G_{0}$ acts semilinearly on $V$ as a $\operatorname{GF}(q)$-vector space, where $q$ is a power of $p$, then we write $G_{0} \leq \Gamma \mathrm{L}_{m}(q)$, where $\Gamma \mathrm{L}_{m}(q)$ is the full semilinear group and $V \simeq \mathrm{GF}(q)^{m}$. If the field is clear from the context, we may use $\Gamma \mathrm{L}(V)=\Gamma \mathrm{L}_{m}(q)$ instead. We write $\mathrm{ALL}_{m}(q)$ for the full affine semilinear group.

Now we are ready to state the classification of affine rank 3 groups.

Theorem 3.1 ([19]). Let $G$ be a finite primitive affine permutation group of rank 3 and degree $n=p^{d}$, with socle $V \simeq \mathrm{GF}(p)^{d}$ for some prime $p$, and let $G_{0}$ be the stabilizer of the zero vector in $V$. Then $G_{0}$ belongs to one of the following classes.

(A) Infinite classes. These are:

(1) $G_{0} \leq \Gamma L_{1}\left(p^{d}\right)$;

(2) $G_{0}$ is imprimitive as a linear group;

(3) $G_{0}$ stabilizes the decomposition of $V \simeq \mathrm{GF}(q)^{2 m}$ into $V=V_{1} \otimes V_{2}$, where $p^{d}=q^{2 m}, \operatorname{dim} V_{1}=2$ and $\operatorname{dim} V_{2}=m ;$

(4) $G_{0} \unrhd \mathrm{SL}_{m}(\sqrt{q})$ and $p^{d}=q^{m}$, where 2 divides $\frac{d}{m}$;

(5) $G_{0} \unrhd \mathrm{SL}_{2}(\sqrt[3]{q})$ and $p^{d}=q^{2}$, where 3 divides $\frac{d}{2}$;

(6) $G_{0} \unrhd \mathrm{SU}_{m}(q)$ and $p^{d}=q^{2 m}$; 
(7) $G_{0} \unrhd \Omega_{2 m}^{ \pm}(q)$ and $p^{d}=q^{2 m}$;

(8) $G_{0} \unrhd \mathrm{SL}_{5}(q)$ and $p^{d}=q^{10}$;

(9) $G_{0} \unrhd B_{3}(q)$ and $p^{d}=q^{8}$;

(10) $G_{0} \unrhd D_{5}(q)$ and $p^{d}=q^{16}$;

(11) $G_{0} \unrhd \mathrm{Sz}(q)$ and $p^{d}=q^{4}$.

(B) 'Extraspecial' classes.

(C) 'Exceptional' classes.

Moreover, classes (B) and (C) consist of finitely many groups.

Observe that the only case when a primitive affine rank 3 group can lie in some other class from the statement of Proposition 2.8 is when it preserves a nontrivial product decomposition. This is precisely case (A2) of the classification, and this situation does occur.

Recall that each rank 3 group gives rise to a rank 3 graph. By [4, Table 11.4], the groups from case (A) of Theorem 3.1 correspond to the following series of graphs:

- One-dimensional affine graphs (i.e. those arising from case (A1)). These graphs are either Van Lint-Schrijver, Paley or Peisert graphs [23];

- Hamming graphs. These graphs correspond to linearly imprimitive groups;

- Bilinear forms graph $H_{q}(2, m)$, where $m \geq 2$ and $q$ is a prime power. These graphs correspond to groups fixing a nontrivial tensor decomposition;

- Affine polar graph $\mathrm{VO}_{2 m}^{\epsilon}(q)$, where $m \geq 2, \epsilon= \pm$, and $q$ is a prime power;

- Alternating forms graph $A(5, q)$, where $q$ is a prime power;

- Affine half spin graph $\mathrm{VD}_{5,5}(q)$, where $q$ is a prime power;

- Suzuki-Tits ovoid graph $\operatorname{VSz}(q)$, where $q=2^{2 e+1}, e \geq 1$.

The reader is referred to [4] for the construction and basic properties of the mentioned graphs.

It should be noted that different cases of Theorem 3.1 may lead to isomorphic graphs. Table 3 lists affine rank 3 groups from case (A) and indicates the corresponding rank 3 graphs. In Tables 1 and 2 we provide degrees and subdegrees of affine rank 3 groups in case (A). These and some other relevant tables and comments on sources of data used are collected in Appendix.

Our first goal is to show that almost all pairs of affine rank 3 graphs can be distinguished based on their subdegrees. We start with the class (A1). The following lemma summarizes some of the arithmetical conditions for the subdegrees of the corresponding groups.

Lemma 3.2. Let $G$ be a primitive affine rank 3 group from class (A1), having degree $n=p^{d}$, where $p$ is a prime. Denote by $m_{1}, m_{2}$ the subdegrees of $G$ and suppose that $m_{1}<m_{2}$. Then $m_{1}$ divides $m_{2}$ and $\frac{m_{2}}{m_{1}}$ divides $d$.

Proof. See [10, Proposition 3.3] for the first claim and [10, Theorem 3.7, (4)] for the second. 
The following lemmas apply conditions from Lemma 3.2 to groups from classes (B), (C) and (A).

Lemma 3.3. Let $G$ be a primitive affine rank 3 group from class (B). Suppose that $G$ has the same subdegrees as a group from class (A1). Then the degree and subdegrees of $G$ are one of the following: $\left(7^{2}, 24,24\right),\left(17^{2}, 96,192\right),\left(23^{2}, 264,264\right),\left(3^{6}, 104,624\right)$, $\left(47^{2}, 1104,1104\right),\left(3^{4}, 16,64\right),\left(7^{4}, 480,1920\right)$.

Proof. Let $n$ denote the degree of $G$, and let $m_{1} \leq m_{2}$ be the subdegrees. In Table 5 all possible subdegrees of groups from class (B) are listed. We apply Lemma 3.2. For instance, if $n=29^{2}$ then $m_{1}=168, m_{2}=672$. The quotient $\frac{m_{2}}{m_{1}}=4$ does not divide 2 , hence this case cannot happen. The other cases are treated in the same manner.

Lemma 3.4. Let $G$ be a primitive affine rank 3 group from class $(C)$. Suppose that $G$ has the same subdegrees as a group from class (A1). Then the degree and subdegrees of $G$ are $\left(3^{4}, 40,40\right)$ or $\left(89^{2}, 2640,5280\right)$.

Proof. Follows from Lemma 3.2 and Table 6.

Lemma 3.5. Let $G$ be a primitive affine rank 3 group from class $(A)$ and suppose that $G$ has the same subdegrees as a group from class (A1). Then either $G$ lies in (A1) or degree and subdegrees of $G$ are one of the following: $\left(3^{2}, 4,4\right),\left(3^{4}, 16,64\right),\left(3^{6}, 104,624\right),\left(2^{4}, 5,10\right)$, $\left(2^{6}, 21,42\right),\left(2^{8}, 51,204\right),\left(2^{10}, 93,930\right),\left(2^{12}, 315,3780\right),\left(2^{16}, 3855,61680\right),\left(5^{2}, 8,16\right)$.

Proof. Suppose that $G$ does not lie in class (A1), but shares subdegrees with some group from (A1). Notice that in cases (A3) through (A11), exactly one of the subdegrees is divisible by $p$, so the subdegrees are not equal (see Table 1). In case (A2) subdegrees are the same if and only if $p^{m}=3$, and consequentially $n=9$. This situation is the first example in our list of parameters, hence from now on we may assume that the subdegrees of $G$ are not equal.

Let $m_{1}$ and $m_{2}$ denote the subdegrees of $G$, where, as shown earlier, we may assume $m_{1}<m_{2}$. Since $m_{1}$ and $m_{2}$ are subdegrees of some group from the class (A1), Lemma 3.2 yields that $m_{1}$ divides $m_{2}$ and the number $u=\frac{m_{2}}{m_{1}}$ divides $d$, where $n=p^{d}$.

Now, since $G$ belongs to one of the classes (A2)-(A11), we apply the above arithmetical conditions in each case. We consider some classes together, since they give rise to isomorphic rank 3 graphs and hence have the same formulae for subdegrees. The reader is referred to Table 1 for the list of subdegrees in question.

(A2) Subdegrees in this case are $2\left(p^{m}-1\right)$ and $\left(p^{m}-1\right)^{2}$. If $2\left(p^{m}-1\right)>\left(p^{m}-1\right)^{2}$, then $p^{m}=2$ and $n=4$. It can be easily seen that $G$ is not primitive in this case, contrary to our hypothesis. Therefore we can assume that $2\left(p^{m}-1\right)<$ $\left(p^{m}-1\right)^{2}$.

Then $u=\frac{p^{m}-1}{2}$ and since $u$ divides $d=2 m$, we have $p^{m}-1 \leq 4 m$. It follows that $\left(n, m_{1}, m_{2}\right)$ is one of $\left(3^{2}, 4,4\right),\left(3^{4}, 16,64\right)$ or $\left(5^{2}, 8,16\right)$.

(A3)-(A5) We write $r$ for the highest power of $p$ dividing $m_{2}$, so the second subdegree is equal to $r\left(r^{m}-1\right)\left(r^{m-1}-1\right)$ for some $m \geq 2$.

We have $u=r \frac{r^{m-1}-1}{r+1}$ and hence $u \geq \frac{r^{m-1}-1}{2}$. Now $r^{2 m}=p^{d} \geq p^{\frac{r^{m-1}-1}{2}}$. Using inequalities $m \geq 2$ and $p \geq 2$, we obtain $2 r^{8(m-1)} \geq 2^{r^{m-1}}$. Therefore 
$r^{m-1} \leq 44$ and there are finitely many choices for $r$ and $m$. Checking these values of $r$ and $m$ against original divisibility conditions we yield the following possibilities for $\left(n, m_{1}, m_{2}\right):\left(2^{6}, 21,42\right),\left(2^{10}, 93,930\right),\left(2^{12}, 315,3780\right)$, $\left(3^{6}, 104,624\right)$.

(A6), (A7) $u=q^{m-1} \frac{q-1}{q^{m-1} \pm 1}$. Numbers $q^{m-1}$ and $q^{m-1} \pm 1$ are coprime, so $q^{m-1} \pm 1$ divides $q-1$. That is possible only when $m=2$, so we have $u=q$. Now $2^{q} \leq p^{q} \leq p^{d}=q^{4}$, so $q \leq 16$. Hence we have the following possibilities for $n, m_{1}, m_{2}$ in this case: $\left(2^{4}, 5,10\right),\left(2^{8}, 51,204\right),\left(2^{16}, 3855,61680\right)$.

(A8) $u=q^{3}-q^{2} \frac{q+1}{q^{2}+1}$. Since $q^{2}$ and $q^{2}+1$ are coprime, $q^{2}+1$ must divide $q+1$. This can not happen, so this case does not occur.

(A9) $u=q^{3} \frac{q-1}{q^{3}+1}$. Since $q^{3}+1$ does not divide $q-1$, this case does not occur.

(A10) $u=q^{5}-q^{3} \frac{q^{2}+1}{q^{3}+1}$. Since $q^{3}+1$ does not divide $q^{2}+1$, this case does not occur.

(A11) $u=q$ and $p^{d}=q^{4}$. Hence we obtain the same possible parameters as in cases (A6), (A7).

In all cases considered we either got a contradiction or got one of the possible exceptions recorded in the statement. The claim is proved.

As an immediate corollary we derive that 2-closures of primitive rank 3 subgroups of $\mathrm{A}_{1}(q)$ also lie in $\mathrm{A}_{1}(q)$ (Theorem 1.2), apart from a finite number of exceptions.

Proof of Theorem 1.2. Suppose that $G$ and $G^{(2)}$ have different socles. Since $G$ is not almost simple, Lemma 2.5 implies that $G^{(2)}$ and thus $G$ must preserve a nontrivial product decomposition. In that situation $G$ has subdegrees of the form $2(\sqrt{n}-1),(\sqrt{n}-1)^{2}$, in particular, $G$ has subdegrees as a group from class (A2) and hence parameters of $G$ are listed in Lemma 3.5. We may assume that $G$ does not preserve a nontrivial product decomposition and so $G$ and $G^{(2)}$ have equal socles. The claim now follows from Theorem 3.1 and Lemmas 3.3-3.5.

Note that Lemmas 3.3-3.5 list degrees and subdegrees of possible exceptions to Theorem 1.2; in Table 7 of Appendix we collect these data in one place.

Now we move on to establish a partial analogue of Lemma 3.5 for classes (A2)-(A11). First we need to recall some notions related to quadratic and bilinear forms.

Let $V$ be a vector space over a field $F$. Given a symmetric bilinear form $f: V \times V \rightarrow$ $F$, the radical of $f$ is $\operatorname{rad}(f)=\{x \in V \mid f(x, y)=0$ for all $y\}$; we say that $f$ is nonsingular, if $\operatorname{rad}(f)=0$. If $\kappa: V \times V \rightarrow F$ is a quadratic form with an associated bilinear form $f$, then the radical of $\kappa$ is $\operatorname{rad}(\kappa)=\operatorname{rad}(f) \cap\{x \in V \mid \kappa(x)=0\}$. We say that $\kappa$ is non-singular, if $\operatorname{rad}(\kappa)=0$, and we say that $\kappa$ is non-degenerate, if $\operatorname{rad}(f)=0$.

If $F$ has odd characteristic, then $\operatorname{rad}(\kappa)=\operatorname{rad}(f)$. If $F$ has even characteristic and $\kappa$ is non-singular, then the dimension of $\operatorname{rad}(f)$ is at most one, $f$ induces a non-singular alternating form on $V / \operatorname{rad}(f)$ and, hence, the dimension of $V / \operatorname{rad}(f)$ is even (see [32, Section 3.4.7]). Therefore if the dimension of $V$ is even, then the notions of non-singular and non-degenerate quadratic forms coincide regardless of the characteristic. 
Now we can describe the affine polar graph $\operatorname{VO}_{2 m}^{\epsilon}(q), m \geq 2$. Let $V$ be a $2 m$ dimensional vector space over $\mathrm{GF}(q)$, and let $\kappa: V \rightarrow \mathrm{GF}(q)$ be a non-singular quadratic form of type $\epsilon$. Vertices of the graph $\mathrm{VO}_{2 m}^{\epsilon}(q)$ are identified with vectors from $V$, and two distinct vertices $u, v \in V$ are joined by an edge if $\kappa(u-v)=0$. Up to isomorphism, $\mathrm{VO}_{2 m}^{\epsilon}(q)$ does not depend on the form $\kappa$.

Allowing some abuse of terminology, we say that subdegrees of a rank 3 graph are simply subdegrees of the respective rank 3 group.

Proposition 3.6. If two affine rank 3 graphs have the same subdegrees, then they are isomorphic apart from the following exceptions:

- graphs arising from affine groups from Table 8 ,

- $\operatorname{VSz}(q)$ and $\mathrm{VO}_{4}^{-}(q)$ for $q=2^{2 e+1}, e \geq 1$,

- Paley and Peisert graphs.

In particular, graphs $H_{q}(2,2)$ and $\mathrm{VO}_{4}^{+}(q)$ are isomorphic.

Proof. Since classes (B) and (C) of Theorem 3.1 and all exceptional parameter sets of Lemma 3.5 are included in Table 8, we may assume that our graphs come from the case (A) and their subdegrees are not among the exceptions from Lemma 3.5.

By Lemma 3.5, if one of the graphs in question arises from the case (A1), then the second graph also comes from (A1). By Table 2, Van Lint-Schrijver graph has unequal subdegrees, while Paley and Peisert graphs have equal subdegrees, hence in this case graphs are either isomorphic or it is a Paley graph and a Peisert graph. We may now assume that our graphs do not come from (A1).

Notice that given $n=p^{d}$ for $p$ prime, the largest subdegree of graphs from classes (A3)-(A11) is divisible by $p$. This is not the case in class (A2), unless $n=4$ with subdegrees 2 and 1 . The corresponding rank 3 group is imprimitive in that situation, contrary to our assumptions. Thus we may assume that none of the two graphs comes from (A2).

We compare subdegrees of classes (A3)-(A11) and collect the relevant information in Table 4. Let us explain the procedure in the case $H_{q}(2, m)$ vs. $\operatorname{VO}_{2 \bar{m}}^{ \pm}(\bar{q})$ only, since other cases are treated similarly.

Consider the graph $H_{q}(2, m)$. The number of its vertices is equal to $n=q^{2 m}$ and the second subdegree is equal to $q\left(q^{m}-1\right)\left(q^{m-1}-1\right)$. Recall that $n=p^{d}$ for some prime $p$, and the largest power of $p$ dividing the second subdegree is $q$. In the case of the graph $\mathrm{VO}_{2 \bar{m}}^{\epsilon}(\bar{q})$, we have $n=\bar{q}^{2 \bar{m}}$ and the largest power of $p$ dividing the second subdegree is $\bar{q}^{\bar{m}-1}$. We obtain a system of equations

$$
q^{2 m}=\bar{q}^{2 \bar{m}}, q=\bar{q}^{\overline{m-1}}
$$

which is written in the relevant cell of Table 4. We derive that $m=\frac{\bar{m}}{\bar{m}-1}$, and hence $m=\bar{m}=2, q=\bar{q}$. Now, the second subdegree for $\operatorname{VO}_{4}^{\epsilon}(q)$ is $q(q-1)\left(q^{2}+(-1)^{\epsilon}\right)$. Therefore $\epsilon=+$, which gives us the first example of affine rank 3 graphs with same subdegrees. Other cases are dealt with in the same way.

Now, Table 4 lists two cases when graphs from different classes have the same subdegrees, namely, $H_{q}(2,2), \mathrm{VO}_{4}^{+}(q)$ and $\mathrm{VSz}(q), \mathrm{VO}_{4}^{-}(q)$. To finish the proof of the proposition, we show that graphs $H_{q}(2,2)$ and $\mathrm{VO}_{4}^{+}(q)$ are in fact isomorphic. 
Identify vertices of $H_{q}(2,2)$ with $2 \times 2$ matrices over $\mathrm{GF}(q)$, and recall that two vertices are connected by an edge if the rank of their difference is 1 . A nonzero $2 \times 2$ matrix has rank 1 precisely when its determinant is zero:

$$
\operatorname{rk}\left(\begin{array}{ll}
u_{1} & u_{3} \\
u_{4} & u_{2}
\end{array}\right)=1 \Longleftrightarrow u_{1} u_{2}-u_{3} u_{4}=0 .
$$

It can be easily seen that $u_{1} u_{2}-u_{3} u_{4}$ is a non-degenerate quadratic form on $\operatorname{GF}(q)^{4}$, so $H_{q}(2,2)$ is isomorphic to the affine polar graph $\mathrm{VO}_{4}^{\epsilon}(q)$. By comparing subdegrees we derive that $\epsilon=+$, and we are done.

It should be noted that $\mathrm{VSz}(q)$ and $\mathrm{VO}_{4}^{-}(q)$ in fact have the same parameters as strongly regular graphs (see [5, Table 24]). In Lemma 3.13 we will see that these graphs are actually not isomorphic since they have non-isomorphic automorphism groups.

Paley and Peisert graphs are generally not isomorphic (see [24]), but have the same parameters since they are strongly regular and self-complementary (i.e. isomorphic to their complements).

Recall that in order to describe 2-closures of rank 3 groups it suffices to find full automorphism groups of corresponding rank 3 graphs. Hamming graphs were dealt with in Proposition 2.4, and graphs arising in the case (A1) were covered in Theorem 1.2. We are left with five cases: bilinear forms graph, affine polar graph, alternating forms graph, affine half spin graph and the Suzuki-Tits ovoid graph. In most of these cases the full automorphism group was described earlier in some form, and we state relevant results here.

For two groups $G_{1}$ and $G_{2}$ let $G_{1} \circ G_{2}$ denote their central product. Note that the central product $\mathrm{GL}(U) \circ \mathrm{GL}(W)$ has a natural action on the tensor product $U \otimes W$.

Proposition 3.7 ([3, Theorem 9.5.1]). Let $q$ be a prime power and $m \geq 2$. Set $G=$ $\operatorname{Aut}\left(H_{q}(2, m)\right)$ and $F=\mathrm{GF}(q)$. If $m>2$, then

$$
G=F^{2 m} \rtimes\left(\left(\mathrm{GL}_{2}(q) \circ \mathrm{GL}_{m}(q)\right) \rtimes \operatorname{Aut}(F)\right) .
$$

If $m=2$, then

$$
G=F^{4} \rtimes\left(\left(\left(\mathrm{GL}_{2}(q) \circ \mathrm{GL}_{2}(q)\right) \rtimes \operatorname{Aut}(F)\right) \rtimes C_{2}\right),
$$

where the additional automorphism of order 2 exchanges components of simple tensors.

Let $V$ be a vector space endowed with a quadratic form $\kappa$. We say that a nonzero vector $v \in V$ is isotropic if $\kappa(v)=0$.

Lemma 3.8 ([27]). Let $V$ be a vector space over some (possibly finite) field $F$, and suppose that $\operatorname{dim} V \geq 3$. Let $\kappa: V \rightarrow F$ be a non-singular quadratic form, possessing an isotropic vector. If $f$ is a permutation of $V$ with the property that

$$
\kappa(x-y)=0 \Leftrightarrow \kappa\left(x^{f}-y^{f}\right)=0,
$$

then $f \in \mathrm{A} \Gamma \mathrm{L}(V)$ and $f: x \mapsto x^{\phi}+v, v \in V$, where $\phi \in \Gamma \mathrm{L}(V)$ is a semisimilarity of $\kappa$, i.e. there exist $\lambda \in F^{\times}$and $\alpha \in \operatorname{Aut}(F)$ such that $\kappa\left(x^{\phi}\right)=\lambda \kappa(x)^{\alpha}$ for all $x \in V$. 
Denote by $\Gamma \mathrm{O}_{2 m}^{\epsilon}(q)$ the group of all semisimilarities of a non-degenerate quadratic form of type $\epsilon$ on the vector space of dimension $2 m$ over the finite field of order $q$. The reader is referred to [17, Sections 2.7 and 2.8] for the structure and properties of groups $\Gamma \mathrm{O}_{2 m}^{\epsilon}(q)$.

Proposition 3.9. Let $q$ be a prime power and $m \geq 2$. Set $F=\operatorname{GF}(q)$. Then

$$
\operatorname{Aut}\left(\mathrm{VO}_{2 m}^{\epsilon}(q)\right)=F^{2 m} \rtimes \Gamma \mathrm{O}_{2 m}^{\epsilon}(q), \epsilon= \pm .
$$

Proof. Recall that the graph $\mathrm{VO}_{2 m}^{\epsilon}(q)$ is defined by a vector space $V=F^{2 m}$ over $F$ and a non-singular (or, equivalently, non-degenerate) quadratic form $\kappa: V \rightarrow F$. Since $m \geq 2$, we have $\operatorname{dim} V \geq 3$ and $\kappa$ possesses an isotropic vector. The claim now follows from Lemma 3.8.

Proposition 3.10 ([3, Theorem 9.5.3]). Let $q$ be a prime power and set $F=\mathrm{GF}(q)$. Then

$$
\operatorname{Aut}(A(5, q))=F^{10} \rtimes\left(\left(\Gamma L_{5}(q) /\{ \pm 1\}\right) \times\left(F^{\times} /\left(F^{\times}\right)^{2}\right)\right) .
$$

Denote by $D_{5}(q)$ an orthogonal group of universal type, in particular, recall the formula $\left|Z\left(D_{5}(q)\right)\right|=\operatorname{gcd}\left(4, q^{5}-1\right)($ see $[7$, Table 5]).

Lemma 3.11. Let $q$ be a prime power, $q^{16}=p^{d}$, let $F=\operatorname{GF}(q), V=F^{16}$ and set $G=\operatorname{Aut}\left(\mathrm{VD}_{5,5}(q)\right)$. Then $G=V \rtimes G_{0}$, and

$$
F^{\times} \circ D_{5}(q) \leq G_{0}=N_{\mathrm{GL}_{d}(p)}\left(D_{5}(q)\right)
$$

where $D_{5}(q)$ acts on the spin module. Moreover, $G_{0} / F^{\times}$is an almost simple group and $G_{0} \leq \Gamma \mathrm{L}_{16}(q)$.

Proof. Set $H=V \rtimes\left(F^{\times} \circ D_{5}(q)\right)$. By [19, Lemma 2.9], $D_{5}(q)$ has two orbits on the set of lines $P_{1}(V)$, so $H$ is an affine rank 3 group of type (A10). Clearly $G=H^{(2)}$ so by Lemma 2.5, $G$ is an affine rank 3 group. By Proposition 3.6, $G$ belongs to class (A10) and the main result of [19] implies that $G_{0} \leq N_{\mathrm{GL}_{d}(p)}\left(D_{5}(q)\right)$. By [19, (1.4)], the generalized Fitting subgroup of $G_{0} / F^{\times}$is simple, hence this quotient group is almost simple. By Hering's theorem [12] (see also [19, Appendix 1]), the normalizer $N_{\mathrm{GL}_{d}(p)}\left(D_{5}(q)\right)$ cannot be transitive on the nonzero vectors of $V$, so $G_{0}=N_{\mathrm{GL}_{d}(p)}\left(D_{5}(q)\right)$ as claimed.

Finally, let $a$ be the minimal integer such that $G_{0} \leq \Gamma \mathrm{L}_{a}\left(p^{d / a}\right)$. By Table $1, a=16$, so the last inclusion follows.

We write Inndiag $\left(D_{5}(q)\right)$ for the overgroup of $D_{5}(q)$ in $\operatorname{Aut}\left(D_{5}(q)\right)$, containing all diagonal automorphisms.

Proposition 3.12. Let $q$ be a prime power, and set $F=\operatorname{GF}(q)$. Then

$$
\operatorname{Aut}\left(\operatorname{VD}_{5,5}(q)\right)=F^{16} \rtimes\left(\left(F^{\times} \circ \operatorname{Inndiag}\left(D_{5}(q)\right)\right) \rtimes \operatorname{Aut}(F)\right) .
$$

Proof. We follow [19, Lemma 2.9]. Take $K=E_{6}(q)$ to be of universal type, so that $|Z(K)|=\operatorname{gcd}(3, q-1)$. The Dynkin diagram of $K$ is:

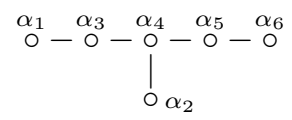


Let $\Sigma$ be the set of roots and let $x_{\alpha}(t), h_{\alpha}(t)$ be Chevalley generators of $K$. Write $X_{\alpha}=$ $\left\{x_{\alpha}(t) \mid t \in F\right\}$. Let $P$ be a parabolic subgroup of $K$ corresponding to the set of roots $\left\{\alpha_{2}, \alpha_{3}, \alpha_{4}, \alpha_{5}, \alpha_{6}\right\}$, and let $P=U L$ be its Levi decomposition. Moreover, $L=M H$ and we may choose $P$ such that

$$
\begin{gathered}
\left.U=\left\langle X_{\alpha}\right| \alpha \in \Sigma^{+}, \alpha \text { involves } \alpha_{1}\right\rangle, \\
M=\left\langle X_{ \pm \alpha_{i}} \mid 2 \leq i \leq 6\right\rangle,
\end{gathered}
$$

where $M$ is of universal type and $H=\left\langle h_{\alpha_{i}}(t) \mid t \in F, 1 \leq i \leq 6\right\rangle$ is the Cartan subgroup. In [19, Lemma 2.9] it was shown that $M \simeq D_{5}(q)$, the group $U$ is elementary abelian of order $q^{16}$ and in fact, it is a spin module for $M$. By [11, Theorem 2.6.5 (f)], $H$ induces diagonal automorphisms on $M$, and by [30, Section 1, B] it induces the full group of diagonal automorphisms. Recall that for an element $h$ of $H$ we have $x_{\alpha}(t)^{h}=x_{\alpha}(k \cdot t)$ for some $k \in F$. In particular, diagonal automorphisms of $D_{5}(q)$ commute with the action of the field $F$ on $U$.

Let $\phi$ be a generator of the field automorphisms group of $K$, and note that one can identify that group with $\operatorname{Aut}(F)$; in particular, $\phi$ acts on $F$ under such an identification. By [11, Theorem 2.5.1 (c)], generators $x_{\alpha}(t)$ and $h_{\alpha}(t)$ are carried to $x_{\alpha}\left(t^{\phi}\right)$ and $h_{\alpha}\left(t^{\phi}\right)$ by $\phi$, so field automorphisms normalize $U, M$ and $H$. Furthermore, $\phi$ induces the full group of field automorphisms on $M$.

Set $T=L \rtimes\langle\phi\rangle$. We have $M \unlhd T$ and $T$ induces all field and diagonal automorphisms on $M$. Set $\bar{T}=T / Z(K)$ and $\bar{M}=M Z(K) / Z(K)$. By [11, Theorem 2.6.5 (e)], the centralizer $C_{\operatorname{Aut}(K)}(U)$ is the image of $Z(U)$ in $\operatorname{Aut}(K)$. Therefore $\bar{T}$ acts faithfully on $U$, and since $|Z(M)|$ is coprime to $|Z(K)|$, we derive that $\bar{M} \simeq M \simeq D_{5}(q)$. Hence we have an embedding $\bar{T} \leq \mathrm{GL}_{d}(p)$, where $|U|=p^{d}$, and, with some abuse of notation, $\bar{T} \leq$ $N_{\mathrm{GL}_{d}(p)}\left(D_{5}(q)\right)$. By Lemma 3.11, the latter normalizer is an almost simple group (modulo scalars), and thus we have shown that it contains all field and diagonal automorphisms of $D_{5}(q)$. It is left to show that it does not contain graph automorphisms.

Suppose that a graph automorphism $\psi$ lies in $G_{0}=N_{\mathrm{GL}_{d}(p)}\left(D_{5}(q)\right)$, and recall that $\bar{M} \simeq D_{5}(q)$. By [19, Lemma 2.9], there is an orbit $\Delta$ of $G_{0}$ on the nonzero vectors of $U$, such that the point stabilizer $\bar{M}_{\delta}, \delta \in \Delta$ is a parabolic subgroup of type $A_{4}$. Since $\psi$ preserves the orbit $\Delta$ and normalizes $\bar{M}$, it must take a point stabilizer $\bar{M}_{\delta}$ to the point stabilizer $\bar{M}_{\delta^{\prime}}$ for some $\delta^{\prime} \in \Delta$, in particular, it takes $\bar{M}_{\delta}$ to a conjugate subgroup. That is impossible, since by [11, Theorem 2.6 .5 (c)], automorphism $\psi$ interchanges conjugacy classes of parabolic subgroups of type $A_{4}$, so the final claim is proved.

Recall the construction of the graph $\operatorname{VSz}(q), q=2^{2 e+1}, e \geq 1$. Set $F=\operatorname{GF}(q)$, $V=F^{4}$ and let $\sigma$ be an automorphism of $F$ acting as $\sigma(x)=x^{2^{e+1}}$. Define the subset $O$ of the projective space $P_{1}(V)$ by

$$
O=\{(0,0,1,0)\} \cap\left\{(x, y, z, 1) \mid z=x y+x^{2} x^{\sigma}+y^{\sigma}\right\}
$$

where vectors are written projectively. The vertex set of $\mathrm{VSz}(q)$ is $V$ and two vectors are connected by an edge, if a line connecting them has a direction in $O$.

Recall that $\mathrm{Sz}(q) \leq \mathrm{GL}_{4}(q)$ is faithfully represented on $P_{1}(V)$ and induces the group of all collineations which preserve the Suzuki-Tits ovoid $O$ (see [14, Chapter XI, Theorem 3.3]). Clearly scalar transformations preserve the preimage of $O$ in $V$, and it can be 
easily seen that $O^{\alpha}=O$ for any $\alpha \in \operatorname{Aut}(F)$. Hence the following group

$$
H=V \rtimes\left(\left(F^{\times} \times \mathrm{Sz}(q)\right) \rtimes \operatorname{Aut}(F)\right)
$$

acts as a group of automorphisms of $\operatorname{VSz}(q)$. By [13, Lemma 16.4.6], $\mathrm{Sz}(q)$ acts transitively on $P_{1}(V) \backslash O$, hence $H$ is a rank 3 group.

We will show that $H$ is the full automorphism group of $\operatorname{VSz}(q)$, but first we need to note the following basic fact.

Lemma 3.13. If $q=2^{2 e+1}, e \geq 1$, then there is no subgroup of $\operatorname{Aut}\left(\mathrm{VO}_{4}^{-}(q)\right)$ isomorphic to $\mathrm{Sz}(q)$. In particular, graphs $\mathrm{VO}_{4}^{-}(q)$ and $\mathrm{VSz}(q)$ are not isomorphic.

Proof. Suppose the contrary, so that $\mathrm{Sz}(q)$ is a subgroup of $\operatorname{Aut}\left(\mathrm{VO}_{4}^{-}(q)\right)$. By Proposition 3.9, we have $\operatorname{Aut}\left(\mathrm{VO}_{4}^{-}(q)\right) \simeq V \rtimes \Gamma \mathrm{O}_{4}^{-}(q)$ for some elementary abelian group $V$. Recall that the orthogonal group $\Omega_{4}^{-}(q)$ is a normal subgroup of $\Gamma \mathrm{O}_{4}^{-}(q)$, and the quotient $\Gamma \mathrm{O}_{4}^{-}(q) / \Omega_{4}^{-}(q)$ is solvable. Clearly $V$ is also solvable, and since $\mathrm{Sz}(q)$ is simple, we obtain an embedding of $\mathrm{Sz}(q)$ into $\Omega_{4}^{-}(q)$. Yet that is impossible, as can be easily seen by inspection of maximal subgroups of $\Omega_{4}^{-}(q)$, see, for instance, [2, Table 8.17]. That is a contradiction, so the first claim is proved.

The second claim follows from the fact that $\mathrm{Sz}(q)$ lies in $\operatorname{Aut}(\operatorname{VSz}(q))$.

Proposition 3.14. Let $q=2^{2 e+1}$, where $e \geq 1$, and set $F=\operatorname{GF}(q)$. Then

$$
\operatorname{Aut}(\operatorname{VSz}(q))=F^{4} \rtimes\left(\left(F^{\times} \times \operatorname{Sz}(q)\right) \rtimes \operatorname{Aut}(F)\right) .
$$

Proof. Let $H=F^{4} \rtimes\left(\left(F^{\times} \times \mathrm{Sz}(q)\right) \rtimes \operatorname{Aut}(F)\right)$ be a rank 3 group acting on $\operatorname{VSz}(q)$ by automorphisms. Set $G=\operatorname{Aut}(\operatorname{VSz}(q))$ and recall that $G=H^{(2)}$. By Lemma 2.5, $G$ is an affine group with the same socle as $H$, and by Proposition 3.6 and Table 3, it follows that $G$ lies in class (A7) or (A11), or it is one of the groups from Table 8. It can be easily checked that there is no group with degree $q^{4}$ and subdegrees $\left(q^{2}+1\right)(q-1), q\left(q^{2}+1\right)(q-1)$ in Table 8, so the last possibility does not happen. By Lemma 3.13, $G$ does not lie in (A7), so it is a group from class (A11). Denote by $H_{0}$ and $G_{0}$ zero stabilizers in $H$ and $G$ respectively. Notice that $H_{0} \leq G_{0}$.

By Theorem 3.1 and Table 1 , we have $G_{0} \leq \Gamma L_{4}(q)$ and $\mathrm{Sz}(q) \unlhd G_{0}$. By [19, (1.4)], given $Z=Z\left(\mathrm{GL}_{4}(q)\right) \simeq F^{\times}$, the generalized Fitting subgroup of $G_{0} /\left(G_{0} \cap Z\right)$ is a simple group. Hence $G_{0} /\left(G_{0} \cap Z\right)$ is an almost simple group with socle $\operatorname{Sz}(q)$.

The outer automorphisms group of $\mathrm{Sz}(q)$ consists of field automorphisms only (see [7, Table 5]), so

$$
\left|G_{0}\right| \leq|Z| \cdot|\operatorname{Aut}(\operatorname{Sz}(q))| \leq\left|F^{\times}\right||\operatorname{Sz}(q)||\operatorname{Aut}(F)| \text {. }
$$

Since $H_{0} \simeq\left(F^{\times} \times \mathrm{Sz}(q)\right) \rtimes \operatorname{Aut}(F)$, the order of $H_{0}$ coincides with the value on righthand side of the inequality. Now $H_{0}=G_{0}$ and the claim is proved.

Proof of Theorem 1.1. Let $G$ be a rank 3 group, and suppose that $G$ is not listed in Table 8. By Proposition 2.8, we may assume that $G$ is a primitive affine group which does not stabilize a product decomposition and, moreover, $G^{(2)}$ is also an affine group. By Theorem 3.1, $G$ is either a one-dimensional affine group (class (A1)), or preserves a bilinear forms graph $H_{q}(2, m), m \geq 2$, an affine polar graph $\mathrm{VO}_{2 m}^{\epsilon}(q), \epsilon= \pm, m \geq 2$, alternating forms graph $A(5, q)$, affine half-spin graph $\mathrm{VD}_{5,5}(q)$, Suzuki-Tits ovoid graph $\mathrm{VSz}(q)$ or lies in class $(\mathrm{B})$ or $(\mathrm{C})$. 
The full automorphism groups of these graphs (i.e. 2-closures of respective groups) are described in Theorem 1.2 (one-dimensional affine groups), Proposition 3.7 (bilinear forms graph), Proposition 3.9 (affine polar graph), Proposition 3.10 (alternating forms graph), Proposition 3.12 (affine half-spin graph) and Proposition 3.14 (Suzuki-Tits ovoid graph). Notice that we do not need to consider classes (B) and (C) as they are included in Table 8 .

Since by Proposition 3.6, the graph $H_{q}(2,2)$ is isomorphic to $\mathrm{VO}_{4}^{+}(q)$, we may exclude it from the bilinear forms case. Now it is easy to see that cases considered in Theorem 1.1 (iv) are mutually exclusive. Indeed, it suffices to prove that graphs from different cases are not isomorphic. By Proposition 3.6, if two affine rank 3 graphs have the same subdegrees, then they belong to the same case except for $\operatorname{VSz}(q)$ and $\mathrm{VO}_{4}^{-}(q), q=2^{2 e+1}$, $e \geq 1$ (note that we group one-dimensional affine graphs into one case). By Lemma 3.13, graphs $\operatorname{VSz}(q)$ and $\mathrm{VO}_{4}^{-}(q)$ are not isomorphic, which proves the claim.

Finally, inclusions of the form $G \leq \mathrm{A \Gamma L}_{a}(q)$ can be read off Table 1 . Notice that in some cases we do not give the minimal value of $a$, for example, if $\mathrm{SU}_{m}(q) \leq G$ lies in class (A6), then $G \leq \mathrm{A}_{m}\left(q^{2}\right)$, but we list the inclusion $G \leq \mathrm{A} L_{2 m}(q)$. This completes the proof of the theorem.

\section{ORCID iDs}

Saveliy V. Skresanov (iD https://orcid.org/0000-0002-8397-5609

\section{References}

[1] E. Bannai, Maximal subgroups of low rank of finite symmetric and alternating groups, J. Fac. Sci. Univ. Tokyo 18 (1972), 475-486.

[2] J. Bray, D. Holt and C. Roney-Dougal, The Maximal Subgroups of the Low-Dimensional Finite Classical Groups, London Mathematical Society Lecture Note Series, Cambridge University Press, 2013, doi:10.1017/cbo9781139192576.

[3] A. E. Brouwer, A. M. Cohen and A. Neumaier, Distance-Regular Graphs, Ergebnisse der Mathematik und ihrer Grenzgebiete. 3. Folge / A Series of Modern Surveys in Mathematics, Springer Berlin Heidelberg, 1989, doi:10.1007/978-3-642-74341-2.

[4] A. E. Brouwer and H. Van Maldeghem, Strongly regular graphs, https://homepages . cwi.nl/ aeb/math/srg/rk3/.

[5] F. Buekenhout and H. Van Maldeghem, A characterization of some rank 2 incidence geometries by their automorphism group, Mitt. Mathem. Sem. Giessen 218 (1994), 1-70.

[6] P. J. Cameron, Finite permutation groups and finite simple groups, Bull. London Math. Soc. 13 (1981), 1-22, doi:10.1112/blms/13.1.1.

[7] J. H. Conway, R. T. Curtis, S. P. Norton, R. A. Parker and R. A. Wilson, Atlas of Finite Groups: Maximal Subgroups and Ordinary Characters for Simple Groups, Clarendon Press, 1985.

[8] S. Evdokimov and I. Ponomarenko, Two-closure of odd permutation group in polynomial time, Discrete Math. 235 (2001), 221 - 232, doi:10.1016/s0012-365x(00)00275-2.

[9] D. A. Foulser, Solvable primitive permutation groups of low rank, Trans. Amer. Math. Soc. 143 (1969), 1-54, doi:10.1090/S0002-9947-1969-0257199-7.

[10] D. A. Foulser and M. J. Kallaher, Solvable, flag-transitive, rank 3 collineation groups, Geom. Dedicata 7 (1978), 111-130, doi:10.1007/bf00181355.

[11] D. Gorenstein, R. Lyons and R. Solomon, The Classification of the Finite Simple Groups, Number 3, Mathematical surveys and monographs, American Mathematical Society, 1994. 
[12] C. Hering, Transitive linear groups and linear groups which contain irreducible subgroups of prime order, II, J. Algebra 93 (1985), 151 - 164, doi:10.1016/0021-8693(85)90179-6.

[13] J. Hirschfeld, Finite Projective Spaces of Three Dimensions, Oxford mathematical monographs, Clarendon Press, 1985.

[14] B. Huppert and N. Blackburn, Finite groups III, number 243 in Grundlehren der mathematischen Wissenschaften, Springer, 1982, doi:10.1007/978-3-642-67997-1.

[15] L. A. Kalužnin and M. H. Klin, On some numerical invariants of permutation groups, Latv. Mat. Ežegod. 18 (1976), 81-99, in Russian.

[16] W. M. Kantor and R. A. Liebler, The rank 3 permutation representations of the finite classical groups, Trans. Am. Math. Soc. 271 (1982), 1-71, doi:10.1090/s0002-9947-1982-0648077-6.

[17] P. B. Kleidman and M. W. Liebeck, The Subgroup Structure of the Finite Classical Groups, London Mathematical Society Lecture Note Series, Cambridge University Press, 1990, doi: 10.1017/cbo9780511629235.

[18] M. H. Klin, On the method of construction of primitive graphs, Trudy NKI 87 (1974), 3-8, in Russian.

[19] M. W. Liebeck, The affine permutation groups of rank three, Proc. London Math. Soc. s3-54 (1987), 477-516, doi:10.1112/plms/s3-54.3.477.

[20] M. W. Liebeck, C. E. Praeger and J. Saxl, On the 2-closures of finite permutation groups, Journal of the London Mathematical Society s2-37 (1988), 241-252, doi:10.1112/jlms/s2-37. 2.241 .

[21] M. W. Liebeck and J. Saxl, The finite primitive permutation groups of rank three, Bull. London Math. Soc. 18 (1986), 165-172, doi:10.1112/blms/18.2.165.

[22] E. M. Luks and T. Miyazaki, Polynomial-time normalizers, Discret. Math. Theor. Comput. Sci. 13 (2011), 61-96, https: / /hal.inria.fr/hal-00990473/.

[23] M. Muzychuk, A classification of one-dimensional affine rank three graphs, Discrete Math. 344 (2021), Paper No. 112400, 3, doi:10.1016/j.disc.2021.112400.

[24] W. Peisert, All self-complementary symmetric graphs, J. Algebra 240 (2001), 209 - 229, doi: 10.1006/jabr.2000.8714.

[25] C. E. Praeger and J. Saxl, Closures of finite primitive permutation groups, Bull. London Math. Soc. 24 (1992), 251-258, doi:10.1112/blms/24.3.251.

[26] C. E. Praeger and C. Schneider, Permutation Groups and Cartesian Decompositions, London Mathematical Society Lecture Note Series, Cambridge University Press, 2018, doi:10.1017/ 9781139194006.

[27] E. M. Schröder, Ein einfacher beweis des satzes von Alexandroff-Lester, J. Geom. 37 (1990), 153-158, doi:10.1007/bf01230368.

[28] S. V. Skresanov, Counterexamples to two conjectures from the Kourovka notebook, Algebra Log. 58 (2019), 249-253, doi:10.1007/s10469-019-09543-1.

[29] J. H. Van Lint and A. Schrijver, Construction of strongly regular graphs, two-weight codes and partial geometries by finite fields, Combinatorica 1 (1981), 63-73, doi:10.1007/bf02579178.

[30] A. V. Vasilyev, Minimal permutation representations of finite simple exceptional groups of types $E_{6}, E_{7}$ and $E_{8}$, Algebra Log. 36 (1997), 302-310, doi:10.1007/bf02671607.

[31] H. W. Wielandt, Permutation groups through invariant relations and invariant functions, The Ohio State University, 1969, doi:doi:10.1515/9783110863383.237.

[32] R. Wilson, The Finite Simple Groups, Graduate Texts in Mathematics, Springer London, 2009, doi:10.1007/978-1-84800-988-2. 


\section{A Appendix}

In this section we collect some relevant tabular data. Table 1 lists information on affine rank 3 groups from class (A), namely, for each group $G$ it provides rough group-theoretical structure (column "Type of $G$ "), degree $n$ and subdegrees. Column " $a$ " gives the smallest integer $a$ such that the stabilizer of the zero vector $G_{0}$ lies in $\Gamma_{a}\left(p^{d / a}\right)$. Most of the information in Table 1 is taken from [19, Table 12], see also [5, Table 10] for the values of $a$.

Table 2 lists the subdegrees of one-dimensional affine rank 3 groups. The first column specifies the type of graph associated to the group in question, next two columns provide degree and subdegrees, and the last column lists additional constraints on parameters involved. By [23], these graphs turn out to be either Van Lint-Schrijver, Paley or Peisert graphs. See [29, Section 2] for the parameters of the Van Lint-Schrijver graph; parameters of Paley and Peisert graphs are computed using the fact that these graphs are self-complementary.

Table 3 lists rank 3 graphs corresponding to rank 3 groups from classes (A1)-(A11), cf. [4, Table 11.4]. Terminology and graph notation is mostly consistent with [4], see also [5, Table 10].

Table 4 records information on when some families of affine rank 3 graphs can have identical subdegrees, the procedure for building this table being described in Proposition 3.6. Trivial cases (when two graphs are the same) are not listed, also graphs from cases (A1) and (A2) are omitted, since they are dealt with separately.

Tables 5 and 6 list degrees and subdegrees of affine rank 3 groups from classes (B) and $(\mathrm{C})$, without repetitions (i.e. parameter sets are listed only once, regardless of whether several groups possess same parameters). If the smaller subdegree divides the largest, the last column gives the respective quotient; otherwise a dash is placed. Information in Table 5 is taken from [9, Theorem 1.1] and [19, Table 13], see also [5, Table 11]. Information in Table 6 before the horizontal line is taken from [10, Theorem 5.3], but notice that we exclude the case of $119^{2}$, since 119 is not a prime number (that error was observed by Liebeck in [19]). Information in Table 6 after the horizontal line is taken from [19, Table 14], with the correction for the case of Alt(9), where subdegrees should be 120, 135 instead of 105, 150, as noted in [5, Table 12].

Table 7 lists parameters of possible exceptions to Theorem 1.2. The table consists of three subtables, corresponding to classes (A), (B) and (C) of Theorem 3.1, i.e. values for the first subtable are taken from Lemma 3.5, for the second from Lemma 3.3, and for the third from Lemma 3.4. Each subtable lists degrees and smallest subdegrees of possible exceptions. Notice that parameters of one-dimensional affine rank 3 groups stabilizing a nontrivial product decomposition are collected in the subtable for the class (A).

Table 8 lists possible exceptions to Theorem 1.1. The first column references the statement where a possible exception first appears, the second column describes the structure of the group, and the third column gives its degree, either explicitly or by referencing another table. Notice that we include classes (B) and (C) of Theorem 3.1 in Table 8; corresponding groups can be found in [19, Table 1 and 2].

Finally, we mention that Tables 7 and 8 list potential exceptions to Theorems 1.2 and 1.1 respectively, in particular, it might be possible to remove some parameter sets and groups by a more careful analysis. 
Table 1: Class (A) in the classification of affine rank 3 groups

\begin{tabular}{|c|c|c|c|c|}
\hline & Type of $G$ & $n=p^{d}$ & $a$ & Subdegrees \\
\hline (A1): & $G_{0}<\Gamma L_{1}\left(p^{d}\right)$ & $p^{d}$ & 1 & See Table 2 \\
\hline (A2): & $G_{0}$ imprimitive & $p^{2 m}$ & $2 m$ & $2\left(p^{m}-1\right),\left(p^{m}-1\right)^{2}$ \\
\hline (A3): & tensor product & $q^{2 m}$ & $2 m$ & $(q+1)\left(q^{m}-1\right), q\left(q^{m}-1\right)\left(q^{m-1}-1\right)$ \\
\hline (A4): & $G_{0} \unrhd \mathrm{SL}_{m}(\sqrt{q})$ & $q^{m}$ & $m$ & $(\sqrt{q}+1)\left(\sqrt{q}^{m}-1\right), \sqrt{q}\left(\sqrt{q}^{m}-1\right)\left(\sqrt{q}^{m-1}-1\right)$ \\
\hline (A5): & $G_{0} \unrhd \mathrm{SL}_{2}(\sqrt[3]{q})$ & $q^{2}$ & 2 & $(\sqrt[3]{q}+1)(q-1), \sqrt[3]{q}(q-1)\left(\sqrt[3]{q}^{2}-1\right)$ \\
\hline (A6): & $G_{0} \unrhd \mathrm{SU}_{m}(q)$ & $q^{2 m}$ & $m$ & $\left\{\begin{array}{l}\left(q^{m}-1\right)\left(q^{m-1}+1\right), q^{m-1}(q-1)\left(q^{m}-1\right), m \text { even } \\
\left(q^{m}+1\right)\left(q^{m-1}-1\right), q^{m-1}(q-1)\left(q^{m}+1\right), m \text { odd }\end{array}\right.$ \\
\hline (A7): & $G_{0} \unrhd \Omega_{2 m}^{\epsilon}(q)$ & $q^{2 m}$ & $2 m$ & $\left\{\begin{array}{l}\left(q^{m}-1\right)\left(q^{m-1}+1\right), q^{m-1}(q-1)\left(q^{m}-1\right), \epsilon=+ \\
\left(q^{m}+1\right)\left(q^{m-1}-1\right), q^{m-1}(q-1)\left(q^{m}+1\right), \epsilon=-\end{array}\right.$ \\
\hline (A8): & $G_{0} \unrhd \mathrm{SL}_{5}(q)$ & $q^{10}$ & 10 & $\left(q^{5}-1\right)\left(q^{2}+1\right), q^{2}\left(q^{5}-1\right)\left(q^{3}-1\right)$ \\
\hline (A9): & $G_{0} \unrhd B_{3}(q)$ & $q^{8}$ & 8 & $\left(q^{4}-1\right)\left(q^{3}+1\right), q^{3}\left(q^{4}-1\right)(q-1)$ \\
\hline (A10): & $G_{0} \unrhd D_{5}(q)$ & $q^{16}$ & 16 & $\left(q^{8}-1\right)\left(q^{3}+1\right), q^{3}\left(q^{8}-1\right)\left(q^{5}-1\right)$ \\
\hline (A11): & $G_{0} \unrhd \mathrm{Sz}(q)$ & $q^{4}$ & 4 & $\left(q^{2}+1\right)(q-1), q\left(q^{2}+1\right)(q-1)$ \\
\hline
\end{tabular}

Table 2: Subdegrees of one-dimensional affine rank 3 groups

\begin{tabular}{cccc}
\hline Graph & Degree & Subdegrees & Comments \\
\hline Van Lint-Schrijver & $q=p^{(e-1) t}$ & $\frac{1}{e}(q-1), \frac{1}{e}(e-1)(q-1)$ & $e>2$ is prime, $p$ is primitive $(\bmod e)$ \\
Paley & $q$ & $\frac{1}{2}(q-1), \frac{1}{2}(q-1)$ & $q \equiv 1(\bmod 4)$ \\
Peisert & $q=p^{2 t}$ & $\frac{1}{2}(q-1), \frac{1}{2}(q-1)$ & $p \equiv 3(\bmod 4)$ \\
\hline
\end{tabular}

Table 3: Rank 3 graphs in class (A)

\begin{tabular}{|c|c|c|c|}
\hline & Type of $G$ & Graph & Comments \\
\hline (A1): & $G_{0}<\Gamma L_{1}\left(p^{d}\right)$ & Van Lint-Schrijver, Paley or Peisert graph & \multirow{11}{*}{$\begin{array}{l}\mathrm{SL}_{m}(\sqrt{q}) \text { stabilizes an } m \text {-dimensional } \\
\text { subspace over } \mathrm{GF}(\sqrt{q}) \\
\mathrm{SL}_{2}(\sqrt[3]{q}) \text { stabilizes a } 2 \text {-dimensional } \\
\text { subspace over } \mathrm{GF}(\sqrt[3]{q})\end{array}$} \\
\hline (A2): & $G_{0}$ imprimitive & Hamming graph & \\
\hline (A3): & tensor product & bilinear forms graph $H_{q}(2, m)$ & \\
\hline (A4): & $G_{0} \unrhd \mathrm{SL}_{m}(\sqrt{q})$ & bilinear forms graph $H_{\sqrt{q}}(2, m)$ & \\
\hline (A5): & $G_{0} \unrhd \mathrm{SL}_{2}(\sqrt[3]{q})$ & bilinear forms graph $H_{\sqrt[3]{q}}(2,3)$ & \\
\hline (A6): & $G_{0} \unrhd \mathrm{SU}_{m}(q)$ & affine polar graph $\mathrm{VO}_{2 m}^{\epsilon}(q), \epsilon=(-1)^{m}$ & \\
\hline (A7): & $G_{0} \unrhd \Omega_{2 m}^{\epsilon}(q)$ & affine polar graph $\mathrm{VO}_{2 m}^{\epsilon}(q)$ & \\
\hline (A8): & $G_{0} \unrhd \operatorname{SL}_{5}(q)$ & alternating forms graph $A(5, q)$ & \\
\hline (A9): & $G_{0} \unrhd B_{3}(q)$ & affine polar graph $\mathrm{VO}_{8}^{+}(q)$ & \\
\hline (A10): & $G_{0} \unrhd D_{5}(q)$ & affine half spin graph $\mathrm{VD}_{5,5}(q)$ & \\
\hline (A11): & $G_{0} \unrhd \mathrm{Sz}(q)$ & Suzuki-Tits ovoid graph VSz $(q)$ & \\
\hline
\end{tabular}

Table 4: Intersections between classes based on subdegrees

\begin{tabular}{|c|c|c|c|c|}
\hline & $\mathrm{VO}_{2 \bar{m}}^{ \pm}(\bar{q})$ & $A(5, \bar{q})$ & $\mathrm{VD}_{5,5}(\bar{q})$ & $\operatorname{VSz}(\bar{q})$ \\
\hline$H_{q}(2, m)$ & $\begin{array}{c}q^{2 m}=\bar{q}^{2 \bar{m}} \\
q=\bar{q}^{m}-1 \\
m=\frac{\bar{m}}{\bar{m}-1} \\
m=\bar{m}=2, q=\bar{q}\end{array}$ & $\begin{array}{c}q^{2 m}=\bar{q}^{10} \\
q=\bar{q}^{2} \\
m=\frac{10}{4} \\
\text { Impossible }\end{array}$ & $\begin{array}{c}q^{2 m}=\bar{q}^{16} \\
q=\bar{q}^{3} \\
m=\frac{8}{3} \\
\text { Impossible }\end{array}$ & $\begin{array}{c}q^{2 m}=\bar{q}^{4} \\
q=\bar{q} \\
m=2 \\
q\left(q^{2}-1\right)(q-1)=q\left(q^{2}+1\right)(q-1) \\
\text { Impossible }\end{array}$ \\
\hline $\mathrm{VO}_{2 m}^{ \pm}(q)$ & & $\begin{array}{c}q^{2 m}=\bar{q}^{10} \\
q^{m-1}=\bar{q}^{2} \\
m=\frac{5}{3} \\
\text { Impossible }\end{array}$ & $\begin{array}{c}q^{2 m}=\bar{q}^{16} \\
q^{m-1}=\bar{q}^{3} \\
m=\frac{8}{5} \\
\text { Impossible }\end{array}$ & $\begin{array}{c}q^{2 m}=\bar{q}^{4} \\
q^{m-1}=\bar{q} \\
m=2, q=\bar{q}\end{array}$ \\
\hline$A(5, q)$ & & & $\begin{array}{c}q^{10}=\bar{q}^{16} \\
q^{2}=\bar{q}^{3} \\
\text { Impossible }\end{array}$ & $\begin{array}{c}q^{10}=\bar{q}^{4} \\
q^{2}=\bar{q} \\
\text { Impossible }\end{array}$ \\
\hline $\mathrm{VD}_{5,5}(q)$ & & & & $\begin{array}{c}q^{16}=\bar{q}^{4} \\
q^{3}=\bar{q} \\
\text { Impossible }\end{array}$ \\
\hline
\end{tabular}


Table 5: Subdegrees of rank 3 groups in class (B)

\begin{tabular}{lll}
\hline$n=p^{d}$ & Subdegrees $m_{1}, m_{2}$ & $\frac{m_{2}}{m_{1}}$ if it is an integer \\
$2^{6}$ & 27,36 & - \\
$3^{4}$ & 32,48 & - \\
$7^{2}$ & 24,24 & 1 \\
$13^{2}$ & 72,96 & - \\
$17^{2}$ & 96,192 & 2 \\
$19^{2}$ & 144,216 & - \\
$23^{2}$ & 264,264 & 1 \\
$3^{6}$ & 104,624 & 6 \\
$29^{2}$ & 168,672 & 4 \\
$31^{2}$ & 240,720 & 3 \\
$47^{2}$ & 1104,1104 & 1 \\
$3^{4}$ & 16,64 & 4 \\
$5^{4}$ & 240,384 & - \\
$7^{4}$ & 480,1920 & 4 \\
$3^{8}$ & 1440,5120 & - \\
\hline
\end{tabular}

Table 6: Subdegrees of rank 3 groups in class (C)

\begin{tabular}{lll}
\hline$n=p^{d}$ & Subdegrees $m_{1}, m_{2}$ & $\frac{m_{2}}{m_{1}}$ if it is an integer \\
$3^{4}$ & 40,40 & 1 \\
$31^{2}$ & $(31-1) \cdot 12,(31-1) \cdot 20$ & - \\
$41^{2}$ & $(41-1) \cdot 12,(41-1) \cdot 30$ & - \\
$7^{4}$ & $\left(7^{2}-1\right) \cdot 20,\left(7^{2}-1\right) \cdot 30$ & - \\
$71^{2}$ & $(71-1) \cdot 12,(71-1) \cdot 60$ & 5 \\
$79^{2}$ & $(79-1) \cdot 20,(79-1) \cdot 60$ & 3 \\
$89^{2}$ & $(89-1) \cdot 30,(89-1) \cdot 60$ & 2 \\
\hline & & \\
$2^{6}$ & 18,45 & - \\
$5^{4}$ & 144,480 & - \\
$2^{8}$ & 45,210 & - \\
$7^{4}$ & 720,1680 & - \\
$2^{8}$ & 120,135 & - \\
$2^{8}$ & 102,153 & - \\
$3^{6}$ & 224,504 & - \\
$7^{4}$ & 240,2160 & 9 \\
$3^{5}$ & 22,220 & 10 \\
$3^{5}$ & 110,132 & - \\
$2^{11}$ & 276,1771 & - \\
$2^{11}$ & 759,1288 & - \\
$3^{12}$ & 65520,465920 & - \\
$2^{12}$ & 1575,2520 & - \\
$5^{6}$ & 7560,8064 & - \\
\hline
\end{tabular}


Table 7: Possible exceptions to Theorem 1.2

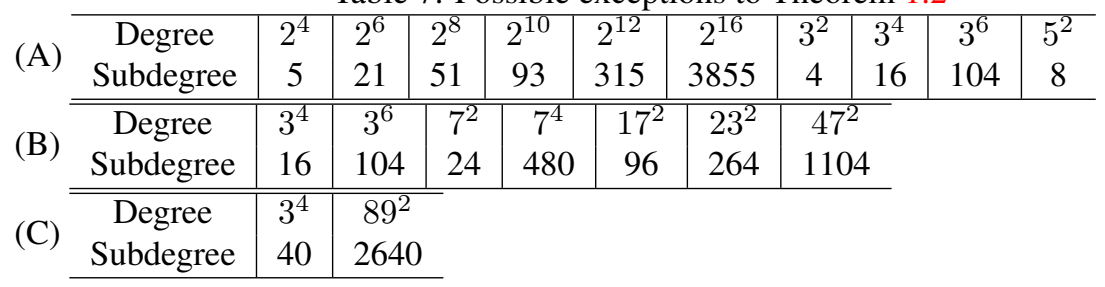

Table 8: Possible exceptions to Theorem 1.1

\begin{tabular}{lll}
\hline Appearance & Type of group & Degree \\
\hline Lemma 2.5 & $\mathrm{P \Gamma L}_{2}(8)$ & 36 \\
& $M_{11}$ & 55 \\
& $M_{12}$ & 66 \\
& $M_{23}$ & 253 \\
& $M_{24}$ & 276 \\
& $\operatorname{Alt}(9)$ & 120 \\
& $G_{2}(q) \unlhd G$ & $q^{3}\left(q^{3}-1\right) / 2$, where $q \in\{3,4,8\}$ \\
& $\Omega_{7}(q) \unlhd G$ & $q^{3}\left(q^{4}-1\right) / \operatorname{gcd}(2, q-1)$, where $q \in\{2,3\}$ \\
Theorem 3.1 & $(\mathrm{B})$ and $(\mathrm{C})$ & Tables 5 and 6 \\
Theorem 1.2 & $G \leq \mathrm{A \Gamma L}(q)$ & Table 7 \\
\hline
\end{tabular}

